\title{
Radiated EMI Modeling and Performance Analysis of a PWM PMSM Drive System Based on Field-circuit Coupled FEM
}

\author{
Youpeng Huangfu ${ }^{1,2}$, Student Member, IEEE, Shuhong Wang ${ }^{1}$, Senior Member, IEEE, \\ Luca Di Rienzo ${ }^{2}$, Senior Member, IEEE, and Jianguo Zhu ${ }^{3}$, Senior Member, IEEE
}

\author{
${ }^{1}$ State Key Laboratory of Electrical Insulation and Power Equipment, School of Electrical Engineering, \\ Xi'an Jiaotong University, Xi'an, 710049, China \\ ${ }^{2}$ Dipartimento di Elettronica, Informazione e Bioingegneria, Politecnico di Milano, Piazza L. da Vinci, 32, Milan 20133, Italy \\ ${ }^{3}$ Faculty of Electrical, Mechanical and Mechatronic Systems, University of Technology Sydney, Sydney, NSW 2007, Australia
}

The radiated electromagnetic interference (EMI) behavior may have a negative impact on the machining accuracy of grinding machines. Therefore, this paper focuses on radiated EMI generated by an ultra-precision pulse width modulation (PWM) permanent magnet synchronous motor (PMSM) drive system. The excitation for the radiated EMI model, the common mode (CM) ground current at the output terminal of the converter, is obtained by simulating its conducted EMI model. The reasonable simplified FE models for the converter frame, the shielded power cable and the motor frame are established. Additionally, an equivalent circuit macro model of the CM impedance of the three-phase PMSM windings is obtained by using impedance measurements, the vector fitting method (VFM) and the fast residue perturbation method (FRPM). Finally, the 3D time harmonic field-circuit coupled finite element method (FEM) is employed to evaluate the radiated EMI.

Index Terms-Field-circuit coupled FEM, common mode current, pulse width modulation, radiated electromagnetic interference.

\section{INTRODUCTION}

$\mathrm{T}$ he machining accuracy of grinding machines is mainly determined by their structural design and the dynamic performance of the electromechanical spindle. However, from the electrical viewpoint, the radiated EMI caused by the PWM motor drive system may have a negative influence on the machining accuracy. Several radiated EMI models for a PWM motor drive system [1]- [2] have been published. Particularly worth mentioning is [1], where the $3 \mathrm{D}$ time harmonic FEM is applied to compute the radiated EMI due to switching frequency. In this paper, the edge current method is used to obtain the equivalent $\mathrm{CM}$ current in each path. The radiated EMI generated by different switching techniques is evaluated with the help of a 3D-FE model [2]. On the other hand, in the following paper the field-circuit coupled FEM is applied.

The considered high-torque ultra-precision PWM drive electromechanical spindle system is used in a computer numerical controlled (CNC) grinding machine. Its machining accuracy could reach the micrometer-level that is suitable for the sophisticated aerospace equipment and national defense manufacturing fields. Unfortunately, it is hypersensitive to the harsh environmental electromagnetic fields.

Thus, to investigate the influence of the radiated EMI,

Manuscript received March 10, 2017; revised April 20, 2017 and June 1, 2015; accepted July 1, 2015. Date of publication July 10, 2015; date of current version July 31, 2015. (Dates will be inserted by IEEE; "published"

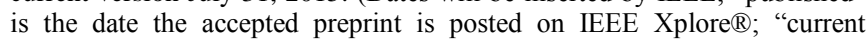
version" is the date the typeset version is posted on Xplore $\left.{ }^{\circledR}\right)$. Corresponding author: Shuhong Wang (e-mail: shwang@mail.xjtu.edu.cn). If some authors contributed equally, write here, "F. A. Author and S. B. Author contributed equally." IEEE TRANSACTIONS ON MAGNETICS discourages courtesy authorship; please use the Acknowledgment section to thank your colleagues for routine contributions.

Color versions of one or more of the figures in this paper are available online at http://ieeexplore.ieee.org.

Digital Object Identifier (inserted by IEEE). firstly the conducted EMI prediction model is established in order to extract the CM ground current, which is then regarded as the excitation for the radiated EMI. In the radiated EMI model the simplified FE models for the converter, the shielded cable and the motor frame are established. An equivalent circuit macro model for the CM impedance of the three-phase windings for PMSM is treated as the load of the cable model.

\section{EXTRACTING THE EXCITATION FOR RADIATED EMI}

Fig. 1 represents the implementation of the radiated EMI prediction procedure. Firstly, the conducted EMI for the drive system is computed by applying MATLAB/Simulink in time domain. Then, the Fast Fourier Transform (FFT) is employed to obtain the $\mathrm{CM}$ ground current excitation for the radiated EMI model in the frequency domain. Finally, such a current is applied to excite the field-circuit coupled FEM model.

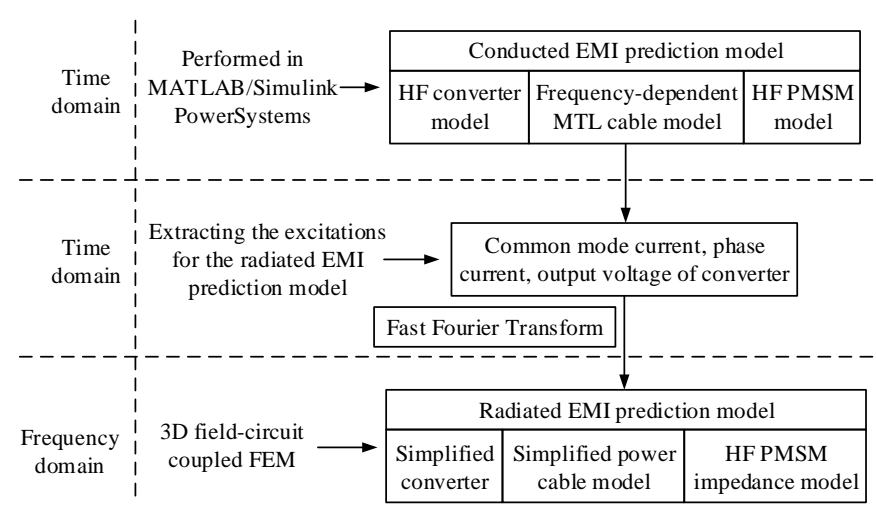

Fig. 1. Implementation procedure for the radiated EMI prediction model.

The diagram for the studied PWM motor drive system is presented in Fig. 2 [3]. It includes a three-phase power supply, a PWM converter (rectifier, DC link and inverter), a shielded 
cable and the PMSM. $I_{\mathrm{CM}}$ is the CM ground current that flows from the output terminal of the converter.

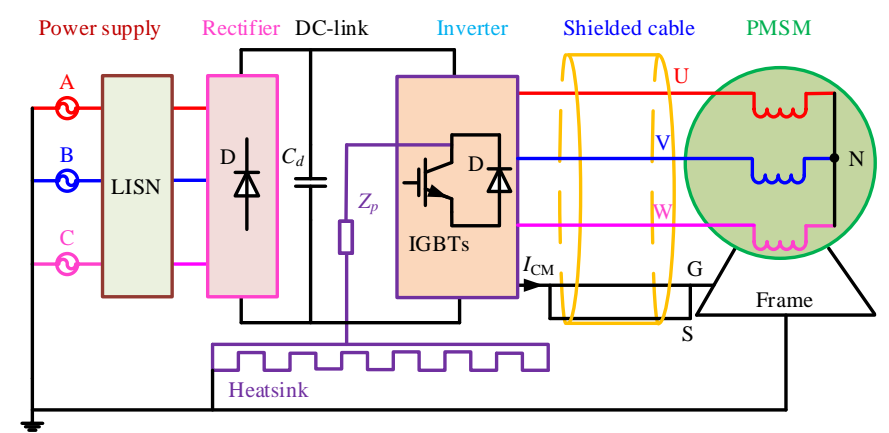

Fig. 2. Description of the PWM motor drive system.

A per-unit-length multi-conductor transmission line (MTL) model of the power cable is analyzed by means of FEM [4]. The high frequency (HF) circuit macro model for PMSM windings is obtained using measurements, VFM and FRPM, respectively [5]- [8]. The CM ground current indicated in Fig. 2 is represented in Fig. 3 by simulating the conducted EMI model. The time range of interest is from $1.56 \mathrm{~ms}$ to $1.62 \mathrm{~ms}$ and the time interval is equals to $2 \mathrm{~ns}$ in order to capture the sparking currents. With the help of the FFT, the amplitude spectrum of the CM ground current is illustrated in Fig. 4. It contains amount of harmonics, thus the time harmonic FEM coupled with circuit model is employed.

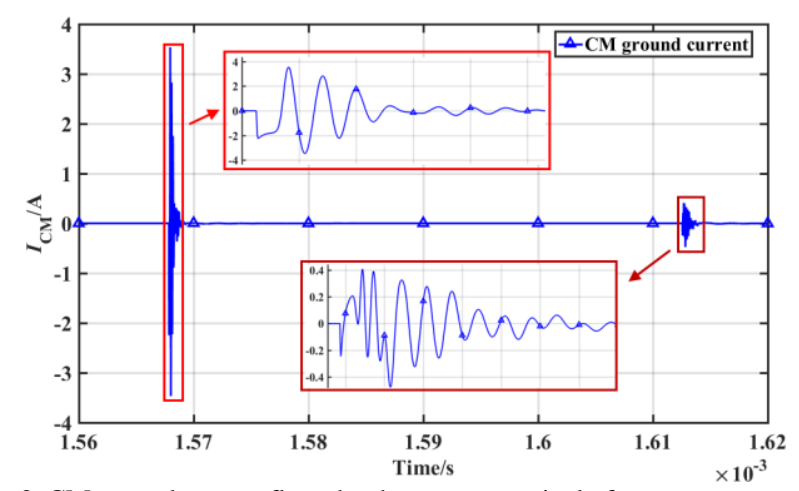

Fig. 3. CM ground current flows by the output terminal of converter.

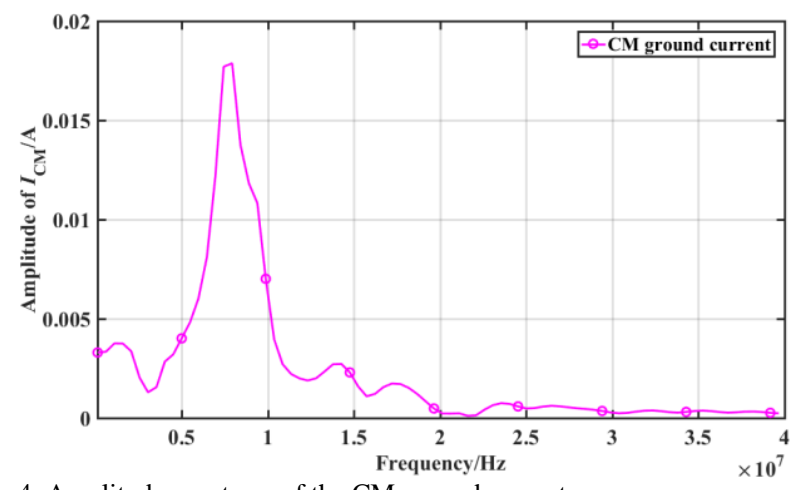

Fig. 4. Amplitude spectrum of the CM ground current.

\section{RADIATED EMI MODEL DESCRIPTION}

\section{A. Overview of the Radiated EMI Model}

The radiated EMI analysis model setup in a short section is schematically demonstrated in Fig. 5.

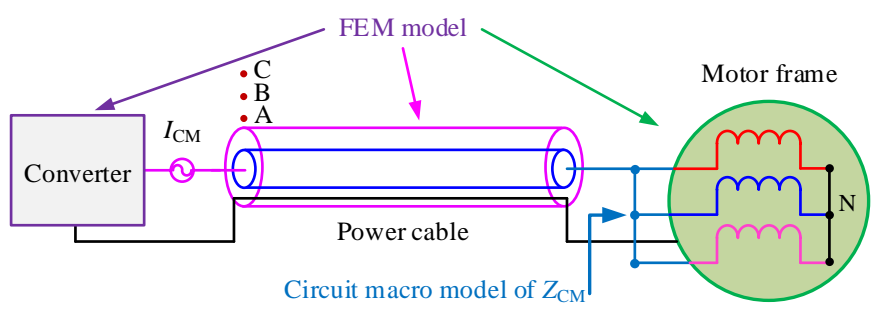

Fig. 5. Schematic for the field-circuit coupled FEM radiated EMI model.

In Fig. 5, the initial terminal of the cable is excited by the $\mathrm{CM}$ ground current. The 3D FEM solves simplified models for the converter, the cable and the motor frame. The circuit macro model $Z_{\mathrm{CM}}$ represents the CM impedance of the PMSM windings. $\mathrm{A}, \mathrm{B}$, and $\mathrm{C}$ are the electromagnetic fields (EMFs) observation points located above the cable at $1 \mathrm{~mm}, 2 \mathrm{~mm}$, and $3 \mathrm{~mm}$, respectively.

\section{B. Simplified FEM Models}

\section{1) Converter Model}

The converter is simplified as a hollow rectangle cavity with a thickness of $3 \mathrm{~mm}$. Its enclosure is made of fire-resistant abs (FR-ABS) resin. Its dimensions and material parameters are presented in TABLE I. Noting that to accelerate the calculation convergence, the conductivity for air and insulation materials are assigned as $1 \mathrm{~S} / \mathrm{m}$.

TABLE I

CONVERTER: DIMENSIONS AND MATERIAL PARAMETERS

\begin{tabular}{llcc}
\hline \hline Item & Quantity & Value & Unit \\
\hline \multirow{3}{*}{ Dimensions } & Height & 474 & $\mathrm{~mm}$ \\
& Width & 222 & $\mathrm{~mm}$ \\
& Depth & 212 & $\mathrm{~mm}$ \\
\hline \multirow{3}{*}{ FR-ABS resin } & Relative permittivity & 4 & - \\
& Relative permeability & 1 & - \\
& Conductivity & 1 & $\mathrm{~S} / \mathrm{m}$ \\
\hline \hline
\end{tabular}

\section{2) Simplified Shielded Power Cable Model}

(a)

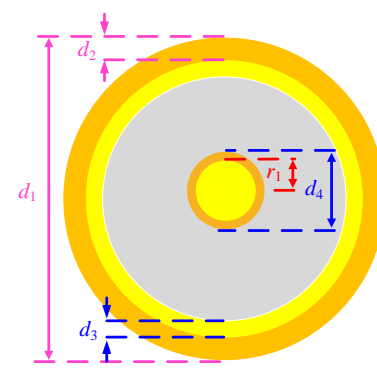

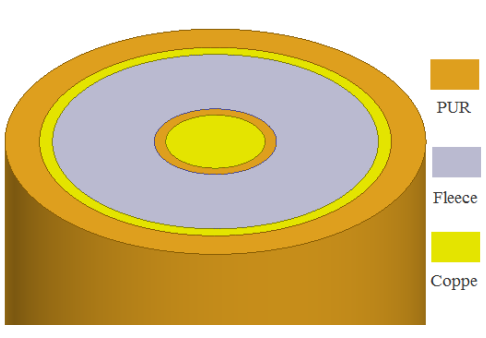

(b)
Fig. 6. (a) Geometrical structure, and (b) material distribution of the cable. 
The real cable includes three-phase wires, ground wire, shield layer and insulation layers. In this work, the CM ground current mainly flows between the ground wire and the shield layer. Moreover, the harmonic component of the CM ground current is much higher than that of the phase currents, so that the radiated EMI greatly depends on the CM ground current. Thus, the cable is modeled simply as the ground wire with the shield layer, as shown in Fig. 6 (a-b). TABLE II shows its dimensions and its material parameters. Obviously, the simplified cable model needs to mesh fewer thin layers than the complete model.

TABLE II

EQUiVALENT CABLE: DIMENSIONS AND MATERIAL PARAMETERS

\begin{tabular}{llcc}
\hline \hline Item & Quantity & Value & Unit \\
\hline \multirow{4}{*}{ Dimensions } & $r_{1}$ & 2.40 & $\mathrm{~mm}$ \\
& $d_{1}$ & 19.50 & $\mathrm{~mm}$ \\
& $d_{2}$ & 1.60 & $\mathrm{~mm}$ \\
& $d_{3}$ & 0.60 & $\mathrm{~mm}$ \\
& $d_{4}$ & 4.20 & $\mathrm{~mm}$ \\
\hline \multirow{3}{*}{ PUR } & Relative permittivity & 3 & - \\
& Relative permeability & 1 & - \\
& Conductivity & 1 & $\mathrm{~S} / \mathrm{m}$ \\
\hline \multirow{3}{*}{ Fleece } & Relative permittivity & 8 & - \\
& Relative permeability & 1 & - \\
& Conductivity & 1 & $\mathrm{~S} / \mathrm{m}$ \\
\hline \multirow{3}{*}{ Cooper } & Relative permittivity & 1 & - \\
& Relative permeability & 1 & - \\
& Conductivity & $5.8 \times 10^{7}$ & $\mathrm{~S} / \mathrm{m}$ \\
\hline \hline
\end{tabular}

\section{3) Simplified Motor Frame Model}

Fig. 7 (a) illustrates the cross section of the simplified motor frame. It consists of a stator and a rotor with two hollow cylinders. TABLE III shows its dimensions and the material parameters.

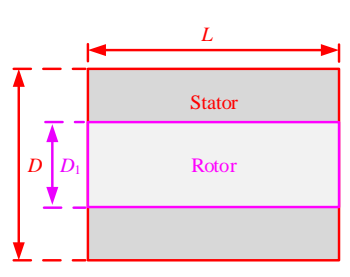

(a)

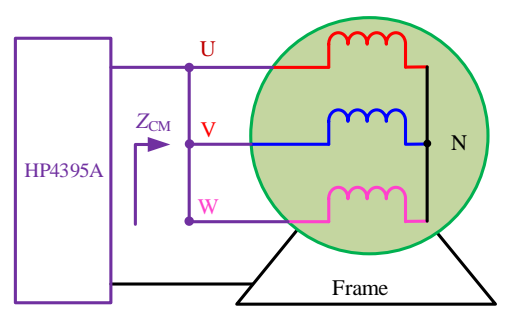

(b)
Fig. 7. (a) Cross section of the simplified motor frame, and (b) CM impedance $Z_{\mathrm{CM}}$ of the three-phase windings of the PMSM.

TABLE III

PMSM FRAME: DIMENSIONS AND MATERIAL PARAMETERS

\begin{tabular}{llcc}
\hline \hline Item & Quantity & Value & Unit \\
\hline \multirow{2}{*}{ Dimensions } & $D$ (Diameter of stator) & 190 & $\mathrm{~mm}$ \\
& $D_{1}$ (Diameter of rotor) & 170 & $\mathrm{~mm}$ \\
& $L$ (Shaft length) & 295 & $\mathrm{~mm}$ \\
\hline \multirow{2}{*}{ Motor } & Relative permittivity & 1 & - \\
Frame & Relative permeability & 8500 & - \\
& Conductivity & $1.2 \times 10^{7}$ & $\mathrm{~S} / \mathrm{m}$ \\
\hline \hline
\end{tabular}

\section{CM Impedance Circuit Macro Model of Motor Windings}

It is difficult to know precisely the winding configurations and geometrical dimensions of the PMSM. Even though, their detailed models would be time and memory consuming. Thus, in order to take into account the influence of the windings, an equivalent circuit macro model for the $\mathrm{CM}$ impedance is introduced. The CM impedance of the three-phase windings of PMSM is defined as the frequency response of the shorted three-phase windings to its frame (ground) [5]. The magnitude and phase of the CM impedance are measured by using the impedance analyzer HP4395A, as presented in Fig. 7 (b).

A high order rational approximation for the $Y(s)=1 / Z_{\mathrm{CM}}$ can be achieved by using the following expansion

$$
Y(s) \cong Y_{\text {rat }}(s)=\sum_{p=1}^{P} \frac{c_{p}}{s-a_{p}}+\sum_{q=1}^{Q}\left(\frac{c_{q}}{s-a_{q}}+\frac{c_{q}^{*}}{s-a_{q}^{*}}\right)+d+s h
$$

where $Y_{\text {rat }}(s)$ is the rational approximation of $Y(s) ; s$ is, the Laplace transform variable; $P$ and $Q$ are the number of real poles and complex conjugate poles pairs, respectively; $a_{p}$ and $c_{p}$ are the real poles and real residues, respectively; $a_{q}$ and $a_{q}{ }^{*}$ are complex conjugate poles pairs; $c_{q}$ and $c_{q}{ }^{*}$ are complex conjugate residues pairs; $d$ and $h$ are constant and proportional terms, respectively.

The unknown variables in (1) can be obtained by employing VFM [6]. The numbers of real poles and complex conjugate poles pairs are 3 and 5 , respectively. Then, the equivalent circuit model for $Z_{\mathrm{CM}}$ is achieved, as shown in Fig. 8. Later, the circuit synthesis [7] is used to obtain its circuit parameters.

However, the system stability of (1) cannot be guaranteed by using VFM. Fortunately, the FRPM [8] can be employed to overcome this problem. Then, the circuit synthesis method is applied again to calculate the circuit parameters, reported in TABLE IV.

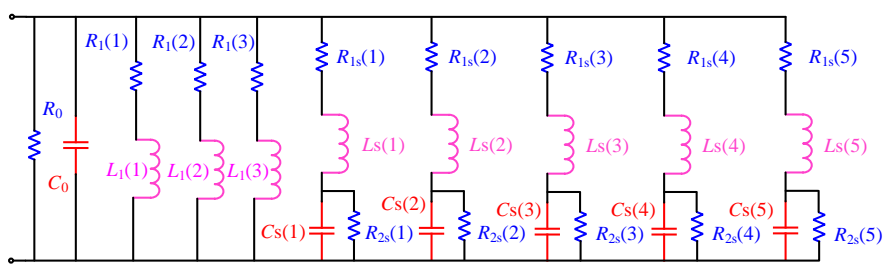

Fig. 8. Equivalent circuit macro model for the $\mathrm{CM}$ impedance $\mathrm{Z}_{\mathrm{CM}}$.

TABLE IV

PARAMETER VAlues For the CirCuit MACRO MOdel OF $Z_{\mathrm{CM}}$

\begin{tabular}{cccc}
\hline \hline$R_{0}(\Omega)$ & $C_{0}(\mathrm{~F})$ & $R_{1}(\Omega)$ & $L_{1}(\mathrm{H})$ \\
\hline & & -102.2429 & $-5.1729 \times 10^{-5}$ \\
45.2601 & $3.5127 \times 10^{-11}$ & 79.2124 & $8.2478 \times 10^{-7}$ \\
& & -34.3761 & $-7.9528 \times 10^{-8}$ \\
\hline \hline$L_{s}(\mathrm{H})$ & $R_{1 s}(\Omega)$ & $C_{s}(\mathrm{~F})$ & $R_{2 s}(\Omega)$ \\
\hline $1.6181 \times 10^{-4}$ & 38.5273 & $2.8573 \times 10^{-9}$ & 1522.4348 \\
$2.2103 \times 10^{-5}$ & -11.1896 & $2.9897 \times 10^{-9}$ & 332.1642 \\
$8.5161 \times 10^{-6}$ & 27.0504 & $1.2488 \times 10^{-9}$ & 4971.2002 \\
$8.1443 \times 10^{-7}$ & 0.1174 & $1.9803 \times 10^{-9}$ & 182.3565 \\
$4.9930 \times 10^{-6}$ & 193.9410 & $9.6699 \times 10^{-12}$ & 6646.3612 \\
\hline \hline
\end{tabular}




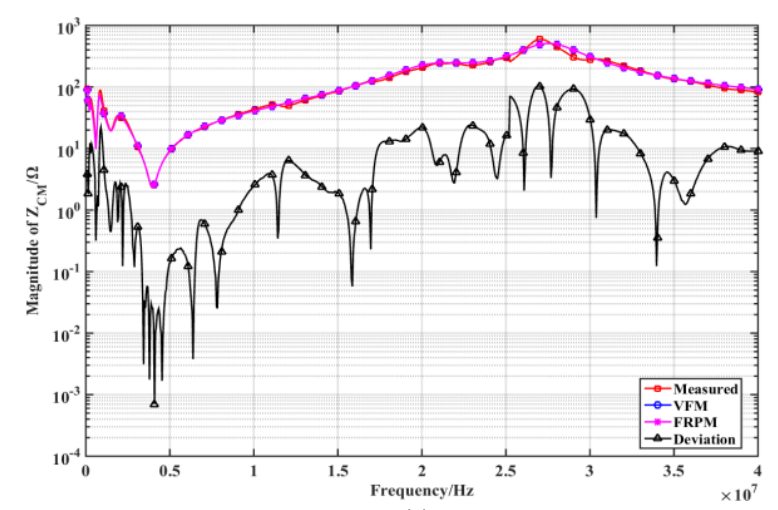

(a)

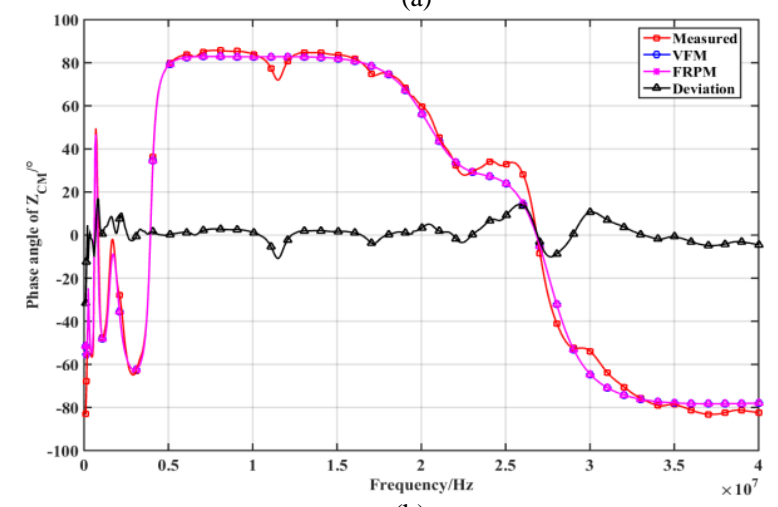

(b)

Fig. 9. Comparisons of $Z_{\mathrm{CM}}$ (a) Magnitude, and (b) phase angle between measurement and VFM and FRPM.

Fig. 9 (a) and (b) represent the magnitude and phase frequency responses for $Z_{\mathrm{CM}}$ based on measurements and given by VFM and FRPM; the agreements are good.

\section{Computation of the Radiated EMI}

The 3D time harmonic FEM coupled with the circuit model is solved using an $\operatorname{Inter}(\mathrm{R}) \mathrm{Xeon}(\mathrm{R})$ E5620 @ $2.4 \mathrm{GHz}$ with 24 GB of memory. The elapsed time is about 50 minutes. Only 82 frequency points in the range of $100 \mathrm{kHz}$ to $40 \mathrm{MHz}$ are computed during the numerical simulation in order to reduce the computational time.

Fig. 10 (a) and (b) illustrate the magnetic flux density and electric field strength of the observation points $\mathrm{A}, \mathrm{B}$, and $\mathrm{C}$.

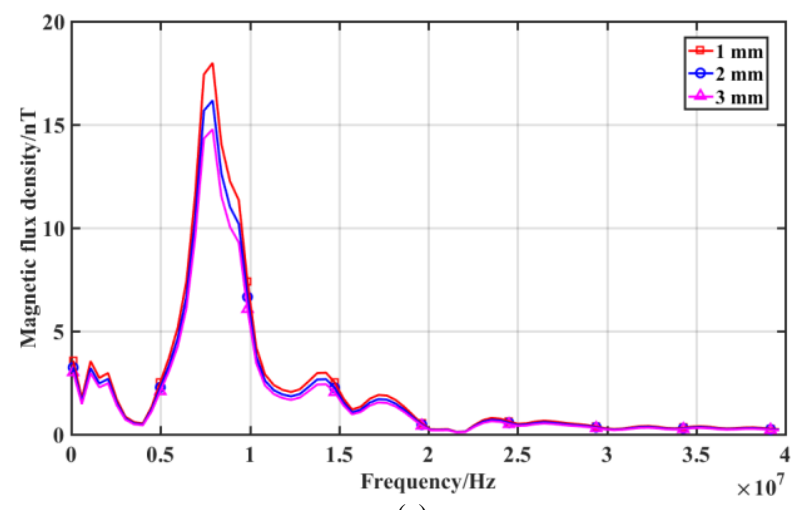

(a)

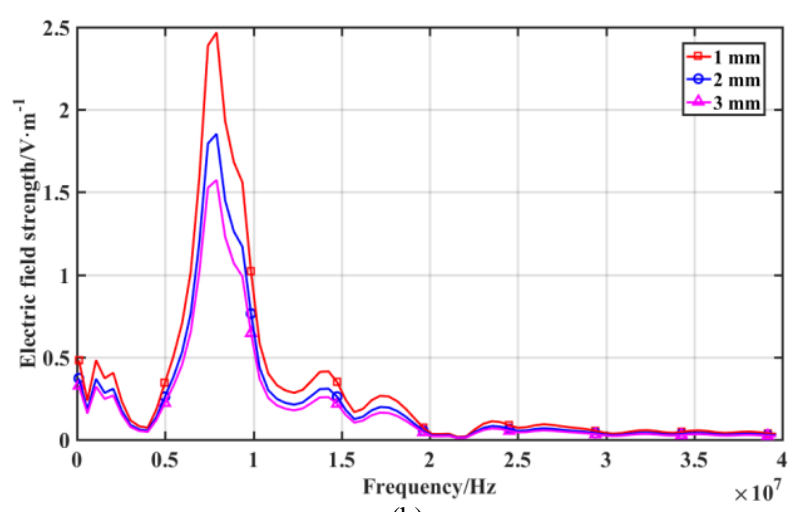

(b)

Fig. 10. (a) Magnetic flux density, and (b) electric field strength at the observation points A, B, and C represented in Fig. 5.

\section{CONCLUSION}

In this paper, a conducted EMI model of a motor drive system is used to predict the radiated EMI. Reasonably simplified 3D FEM model for the devices are employed. The equivalent circuit macro model for the $\mathrm{CM}$ impedance of PMSM windings provides the current path in the circuit model. Then, the 3D time harmonic FEM coupled with the circuit model is applied. The future work will focus on the research of the suppress strategies of radiated EMI.

\section{ACKNOWLEDGMENT}

The authors gratefully acknowledge the support for this research from the National Science and Technology Major Project of China (2014ZX04001-191-01) and Opening Project of State Key Laboratory of Electrical Insulation and Power Equipment at Xi'an Jiaotong University of China (EIPE16201).

\section{REFERENCES}

[1] Andrew Rosales, Ali Sarikhani, and Osama A. Mohammed, "Evaluation of radiated electromagnetic field interference due to frequency switching in PWM motor drives by 3D finite elements," IEEE Trans. Magn., vol. 47, no. 5, pp. 1474-1477, May. 2011.

[2] A. Nejadpak, A. Sarikhani, and O. A. Mohammed, "Analysis of radiated EMI and noise propagation in three-phase inverter system operating under different switching patterns," IEEE Trans. Magn., vol. 49, no. 5, pp. 2213-2216, May. 2013.

[3] Youpeng Huangfu, Shuhong Wang, Juanning Yang, Hongxu Wang, Luca Di Rienzo, and Djilali Hamza, "Conducted EMI simulation for a high power Ultra-precision PMSM driven by PWM converter," 2016 IEEE International Symposium on Electromagnetic Compatibility (EMC), pp: 645-650, Canada, 2016.

[4] Nagy Y. Abed and Osama A. Mohammed, "Frequency-dependent coupled field-circuit modeling of armored power cables using finite elements," IEEE Trans. Magn., vol. 47, no. 5, pp. 930-933, May. 2011.

[5] N. Idir, Y. Weens, M. Moreau, and J. J. Franchaud, "High-frequency behavior models of AC motors," IEEE Trans. Magn., vol. 45, no.1, pp. 133-138, Jan. 2009.

[6] B. Gustavsen and A. Semlyen, "Rational approximation of frequency domain responses by Vector Fitting," IEEE Trans. Power Del., vol. 14, no. 3, pp. 1052-1061, July. 1999.

[7] Giulio Antonini, "SPICE equivalent circuits of frequency-domain responses," IEEE Trans. Electromagn. Compat., vol. 45, no. 3, pp. 502-512, Aug. 2003.

[8] Bjørn Gustavsen, "Fast passivity enforcement for pole-residue models by perturbation of residue matrix eigenvalues," IEEE Trans. Power Del., vol. 23, no. 4, pp. 2278-2285, Oct. 2008. 